\title{
BMJ Open Priority setting partnership to identify the top 10 research priorities for the management of Parkinson's disease
}

\author{
Katherine H O Deane, ${ }^{1}$ Helen Flaherty, ${ }^{1}$ David J Daley, ${ }^{1}$ Roland Pascoe, ${ }^{1}$ \\ Bridget Penhale, ${ }^{1}$ Carl E Clarke,${ }^{2,3}$ Catherine Sackley, ${ }^{4}$ Stacey Storey ${ }^{5}$
}

To cite: Deane KHO,

Flaherty H, Daley DJ, et al. Priority setting partnership to identify the top 10 research priorities for the management of Parkinson's disease. BMJ Open 2014;4: $\mathrm{e} 006434$. doi:10.1136/bmjopen-2014006434

- Prepublication history and additional material is available. To view please visit the journal (http://dx.doi.org/ 10.1136/bmjopen-2014006434).

Received 21 August 2014 Revised 14 November 2014 Accepted 18 November 2014

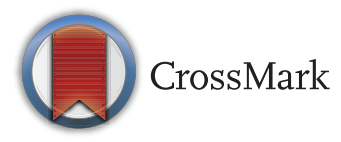

${ }^{1}$ School of Health Sciences, University of East Anglia, Norwich, UK

${ }^{2}$ School of Clinical and Experimental Medicine, College of Medical and Dental Sciences, University of Birmingham, Birmingham, UK

${ }^{3}$ Department of Neurology, Sandwell and West Birmingham Hospitals NHS Trust, City Hospital, Birmingham, UK

${ }^{4}$ Kings College, London, UK

${ }^{5}$ Parkinson's UK, London, UK

Correspondence to Dr Katherine HO Deane; k.deane@uea.ac.uk

\section{ABSTRACT}

Objectives: This priority setting partnership was commissioned by Parkinson's UK to encourage people with direct and personal experience of the condition to work together to identify and prioritise the top 10 evidential uncertainties that impact on everyday clinical practice for the management of Parkinson's disease (PD).

Setting: The UK.

Participants: Anyone with experience of PD including: people with Parkinson's (PwP), carers, family and friends, healthcare and social care professionals. Non-clinical researchers and employees of pharmaceutical or medical devices companies were excluded. 1000 participants (60\% PwP) provided ideas on research uncertainties, 475 (72\% PwP) initially prioritised them and 27 (37\% PwP) stakeholders agreed a final top 10 .

Methods: Using a modified nominal group technique, participants were surveyed to identify what issues for the management of PD needed research. Unique research questions unanswered by current evidence were identified and participants were asked to identify their top 10 research priorities from this list. The top 26 uncertainties were presented to a consensus meeting with key stakeholders to agree the top 10 research priorities.

Results: 1000 participants provided 4100 responses, which contained 94 unique unanswered research questions that were initially prioritised by 475 participants. A consensus meeting with 27 stakeholders agreed the top 10 research priorities. The overarching research aspiration was an effective cure for PD. The top 10 research priorities for PD management included the need to address motor symptoms (balance and falls, and fine motor control), non-motor symptoms (sleep and urinary dysfunction), mental health issues (stress and anxiety, dementia and mild cognitive impairments), side effects of medications (dyskinesia) and the need to develop interventions specific to the phenotypes of PD and better monitoring methods.

Conclusions: These research priorities identify crucial gaps in the existing evidence to address everyday practicalities in the management of the complexities of PD.

\section{Strengths and limitations of this study}

- A key strength of this priority setting partnership was that 1000 people with Parkinson's, their carers and healthcare and social care professionals identified everyday issues which currently lack firm evidence to direct their management.

- In total, 475 participants prioritised these uncertainties, and 27 key stakeholders agreed the top 10 research priorities for the management of Parkinson's disease during a final consensus meeting.

- Very few participants were from minority ethnic populations or living in care homes which could limit the generalisability of these priorities to these populations.

- The top 10 research priorities for Parkinson's disease management were identified by a wide range of people affected by the disease. These included the need to address motor symptoms, non-motor symptoms, mental health issues, side effects of medications and the need to develop interventions specific to the phenotypes of Parkinson's disease and better monitoring methods.

- It is hoped that this top 10 will lead to future research that will address issues of importance for the clinical management of Parkinson's disease.

\section{INTRODUCTION}

Ensuring that research is effective in addressing the needs of patients and the clinicians treating them is critically important. The research agenda has been accused of being overly influenced by the pharmaceutical and medical devices industries, ${ }^{1-3}$ and of not addressing the questions about treatments that are of greatest importance to patients, their carers and clinicians. ${ }^{4-6}$ Research needs to focus on whether treatments are doing more harm than good, or whether one treatment is better than another, and ensure the outcomes reflect issues that have impact on the patient's well-being and participation. ${ }^{7-9}$ 
Treatment uncertainties are defined as questions about the effectiveness of treatments which are not adequately answered by systematic reviews of existing research evidence. ${ }^{10}$ The James Lind Alliance ${ }^{11}$ (JLA) was established to encourage collaboration between patients and clinicians to ensure that uncertainties that impact on everyday clinical practice are addressed in research. One of JLA's approaches is priority setting partnerships ${ }^{12}$ which aim to identify the top 10 research priorities in a given area from the perspectives of patients, carers and healthcare professionals. These research priorities can then inform research funding strategies and policies. ${ }^{13}$

A number of research funders have indicated that they wish to incorporate the findings of priority setting partnerships into their prioritisation processes. ${ }^{13}$ Parkinson's $\mathrm{UK}^{14}$ identified four priority research areas with the specific aim to find a cure for Parkinson's disease (PD). This project expands this initial work and identifies the top 10 research priorities for the management of PD.

\section{METHODS}

The project was led by Parkinson's UK, with the University of East Anglia and the University of Birmingham acting as academic partners. The JLA provided an independent chair, advised on the methodology, and facilitated the process. The project was instigated by Parkinson's UK's Research Support Network ${ }^{15}$ who tasked the steering group to oversee the project.

\section{Ethics and data protection}

We took advice from the National Research Ethics Service ${ }^{16}$ who advised that priority setting partnerships are service evaluations and therefore did not need approval from an NHS ethics committee. The reasoning was that we were asking about research preferences and research is a 'standard' part of NHS treatment protocols. ${ }^{17}$ Therefore, the project recruited participants via multiple routes including direct from the NHS. It was assumed that any participants able to complete the survey had sufficient mental competence to take part in the project. Our safeguarding expert (BP) provided advice on any responses that raised concerns in relation to the responses provided.

Participants could answer the initial survey anonymously. Participants who provided their contact details were recontacted for the prioritisation survey. Respondents' personal details were kept by Parkinson's UK in line with the Data Protection Act, and the UEA team were provided with an anonymised database of responses.

\section{The priority setting partnership stages}

This method is summarised in figure 1 .

\section{Initiation}

The first stage involved the identification of potential partner organisations which provided access to a wide range of participants. Anyone living in the UK with experience and understanding of living with PD was eligible to participate in the identification of uncertainties and their prioritisation. This included: people with Parkinson's ( $\mathrm{PwP})$; carers and former carers; family members and friends; healthcare and social care professionals who work, or have worked, with people living with the condition. Non-clinical researchers and employees of pharmaceutical or medical devices companies were excluded from the survey.

\section{Consultation}

The steering group had a broad spectrum of representatives and they identified the scope of the priority setting partnership. All aspects of the management of PD such as healthcare, surgery, rehabilitation, medication, complementary therapies, nutrition, carer support, service provision and design were included. Excluded issues were curative therapies, prevention, diagnostic tests, aetiology, epidemiology and prognosis. Curative and preventive therapies were agreed to be those that halted or reversed the neurodegenerative processes seen in PD. It was agreed that the overarching research aspiration was an effective cure for PD, and that the specifics of how this could be addressed had been identified previously by Parkinson's UK. ${ }^{14}$

A simple survey was created that asked about four areas where respondents would like to see issues answered by research: the symptoms of PD; day-to-day life with PD; the treatment of PD; anything else.

The exact phrasing of the survey was refined after a pilot survey involving $57 \mathrm{PwP}$ and 20 carers, in order to ensure clarity of meaning and encourage the generation of relevant responses. A copy of the final version of the survey is provided in online supplementary material.

Participants were invited to complete the survey on the Parkinson's UK website or by post. Parkinson's UK advertised the study in their membership magazine which goes out to more than 35000 people affected by Parkinson's, on their website and targeted relevant groups at meetings and conferences including: 13 Parkinson's UK regional events, PD Nurse Specialists Association conference 2013, British Movement Disorders Group (BritMODIS) Conference 2014, National Parkinson's UK Research Supporters Conference 2013, Oxford Parkinson's Disease Centre Open Day 2013, South West Research Supporters day (Bristol), North West information day (St Helens). Parkinson's UK also advertised the project directly to centres of clinical excellence throughout the country, the Tracking Parkinson's centres ${ }^{18}$ and the Oxford Parkinson's Disease Centre.

We contacted relevant charities such as Cure Parkinson's UK, the Alzheimer's Society and the Alzheimer's Research Trust who promoted participation of their members via flyers, magazine articles and social media. We contacted professional groups with an interest in PD such as The British Geriatrics Society, The Specialist Section for Neurological Practice at the College 
Figure 1 Flow chart of methods (F\&F, family and friends; HSCP, health and social care professionals; PwP, people with Parkinson's; PICO, population, intervention, comparator and outcome).

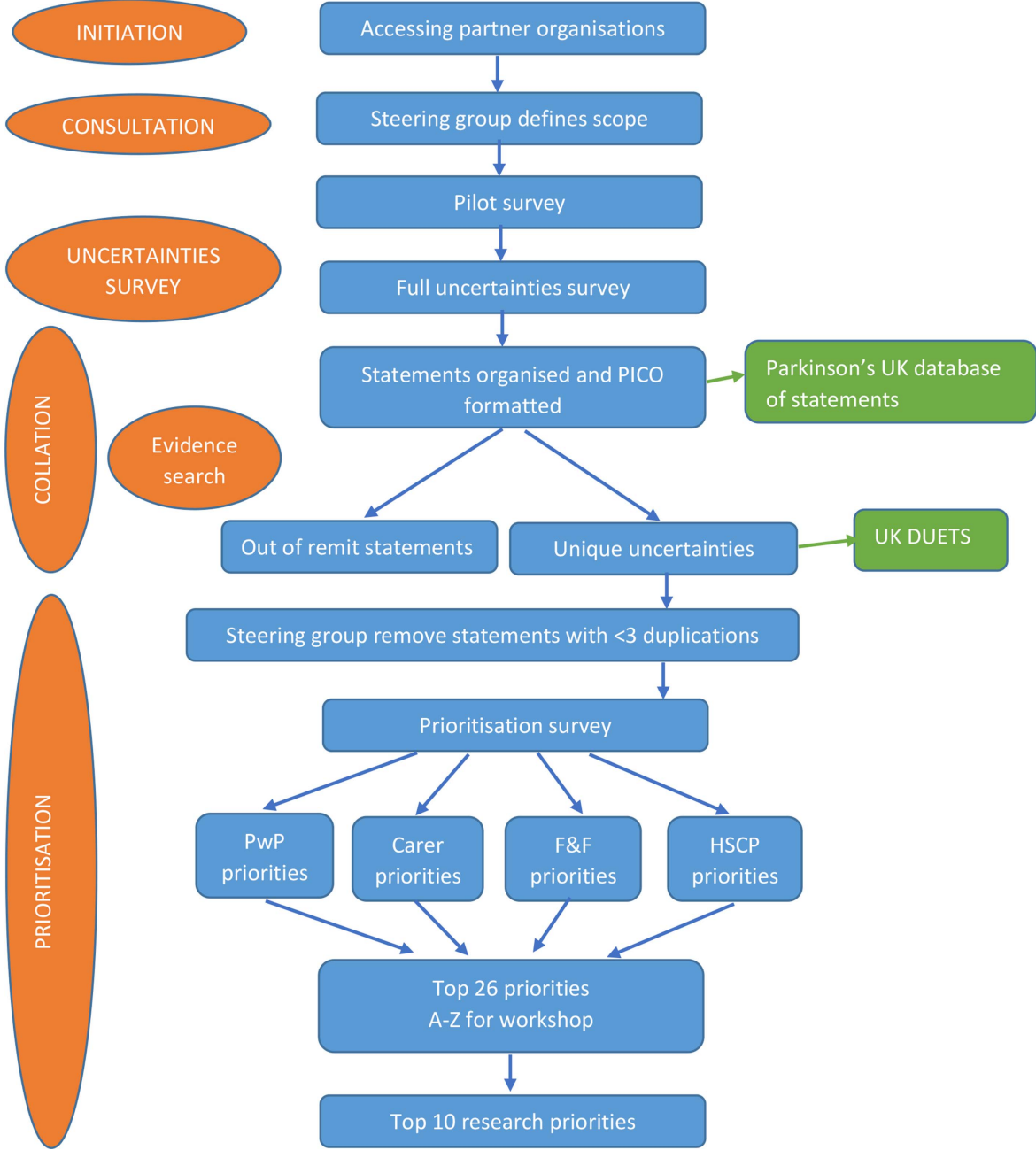

of Occupational Therapists, PD Specialist Interest Group of the Royal College of Speech and Language Therapists and the Parkinson's Nurse Specialists Association. We contacted the National Institute for Health Research's Dementias and Neurodegeneration (DeNDRoN) ${ }^{19}$ Specialty Clinical Research Network and the Enabling Research In Care Homes group (ENRICH) ${ }^{20}$ in order to ask them to encourage participation in the survey by neurological clinicians and the care home sector and residents. We used Parkinson's UK's database of people from black and minority ethnic populations who have an interest in PD to enhance our outreach to these communities. When sending out the survey we noted that we could take responses over the phone and provide a translation service if needed. Representatives from Parkinson's UK visited a couple of movement disorder clinics to promote the survey.

\section{Collation}

The 'raw' treatment uncertainties were entered onto the database verbatim.

Statements not associated with management uncertainties were coded as being 'out of remit'. In order to maximise the value of respondents' comments we also coded 'treatment dissatisfactions' where we were provided with information regarding poor treatment provision. These were shared with Parkinson's UK's Policy and Service Improvement and Professional Engagement and Education departments in order for them have anonymised anecdotes to demonstrate the impact of poor care on PwP.

The Cochrane Library, MEDLINE, EMBASE, CINAHL, PsychInfo were searched from inception to January 2014 for systematic reviews of interventions for the management of Parkinson's. The major systematic review's certainties were agreed by the core team (KHOD, CEC, CS, DJD, RP and HF) before the submissions were received. However, all submissions were checked subsequently against the evidence base to determine if there were any further certainties in unanticipated areas. Submissions which related to management certainties were labelled as 'evidence found' and forwarded to Parkinson's UK as this assisted in the identification of a lack of knowledge of effective treatments, and so demonstrated a need for education.

In order to standardise the format each uncertainty was transformed into population, intervention, comparator and outcome (PICO) format by the coding team (KHOD, DJD, RP and HF). The coding team met fortnightly to discuss coding issues and ensure consistency. 
KHOD double checked the coding of the majority of the statements to ensure consistency. Respondents were able to provide multiple statements in response to each of the four prompts (the symptoms of PD; day-to-day life with PD; the treatment of PD; anything else). Frequently we derived more than one PICO question from a single statement. The PICO questions were expressed as individual research questions, which were then assembled and duplicates combined. The frequency of duplicated uncertainties was recorded. The resulting PICO questions were checked again against the evidence base by KHOD, CEC and CS.

\section{Prioritisation}

The steering group met to examine the uncertainties identified. Uncertainties were checked for specificity in order that they could be adequately investigated and were phrased in a manner understandable to participants. Uncertainties with less than three duplicate submissions were considered for exclusion. This was in order to reduce the number of uncertainties for prioritisation to a manageable level, and is recommended by the JLA's methodology. ${ }^{12}$

The final set of uncertainties were then sent to participants who had provided their contact details and to the members of the Research Support Network. ${ }^{15}$ It was also advertised in an article in the Parkinson's UK membership magazine and promoted through social media. Participants were asked to read the list of uncertainties and identify their own top 10 priorities. A copy of the survey is provided in online supplementary materials. The ratings for each uncertainty statement were scored in reverse, that is, a priority rated first would have 10 points assigned. These were then summated and divided by the number of respondents from each group of participants (PwP, carers, family and friends, and professionals) and ranked in order of priority. These four sets of ratings were then added together and again ranked to identify the top 26 for all participants. The top 26 uncertainties were chosen because the experience of the $\mathrm{JLA}^{12}$ is that this is a sufficient number of uncertainties to identify the top 10 , and in order to be able to label them A-Z.

The final consensus meeting to agree the top 10 research priorities involved participants from a range of populations impacted by PD and used consensusreaching decision-making methods. Prior to the meeting participants were asked to prioritise the top 26 uncertainties which were presented in random order and labelled A-Z.

At the final workshop, ground rules were agreed about keeping participants' opinions and disclosures confidential to the workshop participants alone and respecting alternative viewpoints. The process' intent was to identify a set of prioritised recommendations while preventing the domination of the discussions by a single person and encouraging quieter group members to participate. Expectations were managed by highlighting that consensus meant that people were unlikely to leave the meeting with all of their views being represented in the top 10 , and that compromise would be necessary.

Participants were divided into three groups with mixed representation and led by an independent chairperson from JLA and two facilitators from Parkinson's UK. Participants were asked to prioritise all 26 uncertainties which were printed onto A4 cards. Where there was disagreement about the level of priority, each card had the interim prioritisation for each group ( $\mathrm{PwP}$, carers, family and friends, and professionals) written on the back, and this information assisted decisions about rankings. The three sets of rankings were combined. Participants were then divided into three different groups, again with mixed representation. In the second round they were presented with the joint prioritisation from round one and focused mainly on identifying whether the correct uncertainties were in the top 10 , and had the right prioritisation. The three groups' prioritisations were combined and the final top 15 presented in a final round to the entire group. The final group was reminded that all of the uncertainties presented had insufficient evidence at present to inform clinical practice. In the final round participants reached consensus on which items should be in the top 10 .

\section{Databases}

The complete list of all of the uncertainties identified was prepared and formatted for inclusion in the UK Database of Uncertainties about the Effects of Treatments (UK DUETs). ${ }^{10}$ This will allow researchers to examine the all of the research uncertainties identified in this project.

A searchable database of the anonymised responses of the participants regarding their concerns about the management of PD will be made available to researchers via Parkinson's UK. This will allow researchers to include quotes that highlight the impact of a particular issue on $\mathrm{PwP}$ in their grant applications and research.

\section{RESULTS}

\section{Steering group}

The project's steering group consisted of representatives from Parkinson's UK $(n=8)$, and the Cure Parkinson's Trust $(n=1), \operatorname{PwP}(n=2)$, carers $(n=2)$, clinical consultants $(n=2)$ and a PD nurse specialist $(n=1)$. Those from Parkinson's UK included representatives with expertise in research development, policy and campaigns $(n=5)$, information and support worker services $(n=1)$, advisory services $(n=1)$ and resources and diversity $(n=1)$.

\section{Consultation}

Respondents could provide more than one uncertainty for each of the four areas asked about (the symptoms of PD; day-to-day life with PD; the treatment of PD; anything else). Hence 1000 participants generated 4100 
Figure 2 Flow chart of responses.

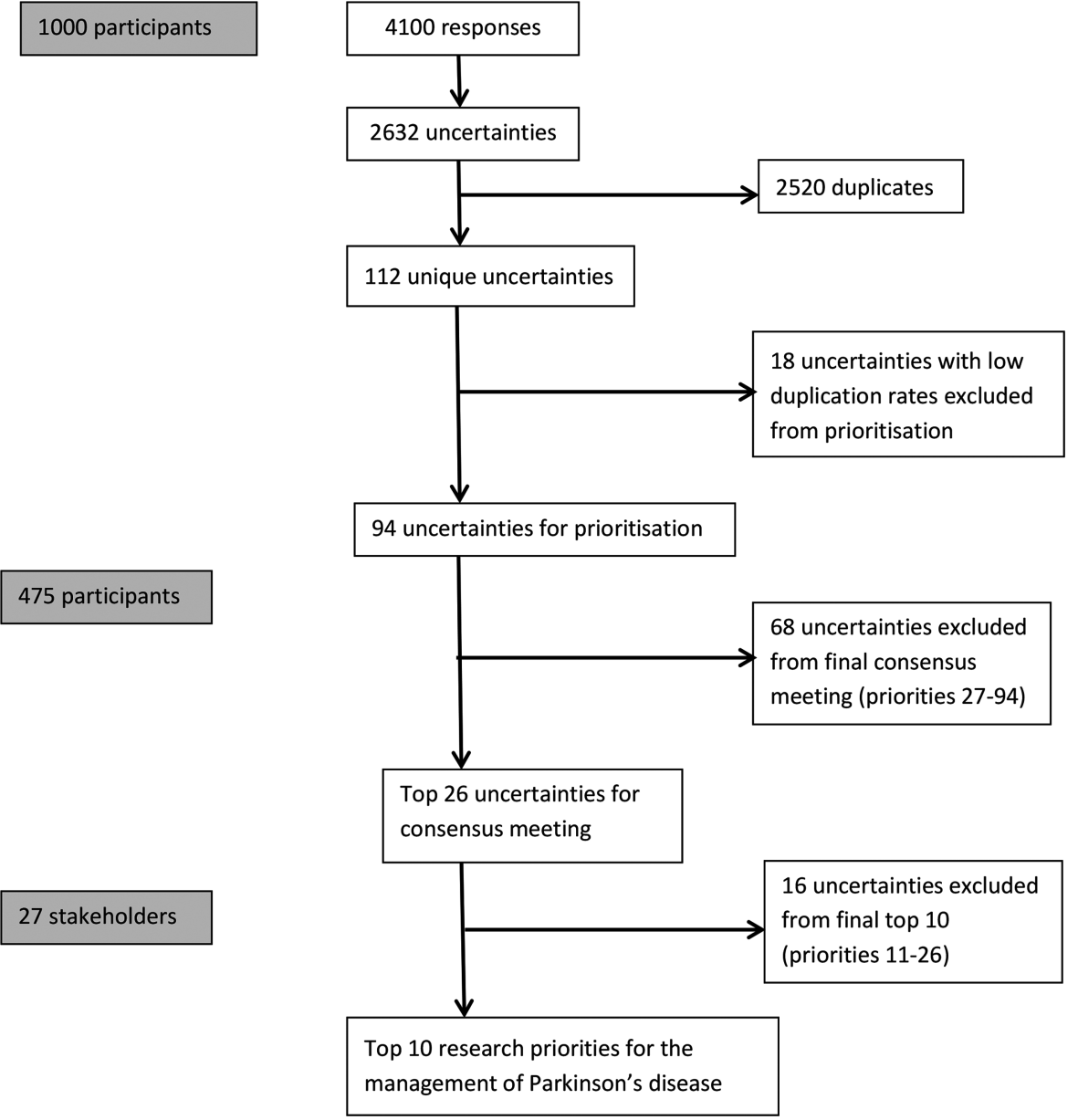

responses which contained 2632 research uncertainties, of which 112 were unique (figure 2). Around $40 \%$ of responses were returned by post $(n=397)$, the remainder were submitted online. No one used the translation service, but a representative from Parkinson's UK did assist some $\mathrm{PwP}$ recruited in movement disorders clinics to complete the form when they had limited English literacy.

The 600 respondents with PD mostly lived at home either with support from carers or family $(51 \%)$, or independently $(41 \%), 1 \%$ lived in nursing or residential accommodation (the remainder were in other accommodation or did not respond; table 1). Professionals

\begin{tabular}{llll} 
Table 1 & Table of participant characteristics & \\
\hline & $\begin{array}{l}\text { People with } \\
\text { Parkinson's }\end{array}$ & Carers & $\begin{array}{l}\text { Family and } \\
\text { friends }\end{array}$ \\
\hline Number & 600 & 136 & 86 \\
Median age range & $65-74$ & $65-74$ & $55-64$ \\
Ethnicity (\%) & & & \\
$\quad$ White & 86 & 90 & 90 \\
Black or Asian & 5 & 1 & 7 \\
Other & 2 & 1 & 2 \\
Not stated & 7 & 8 & 1 \\
\hline
\end{tabular}

$(\mathrm{n}=140)$ consisted of consultants $(24 \%)$, PD nurse specialists $(19 \%)$, nurses and care assistants $(9 \%)$, allied health professionals $(31 \%)$, social workers $(1 \%)$ and others (16\%). Thirty-one respondents classified themselves as 'other' and seven respondents did not provide information on their role.

\section{Research certainties}

The academic team agreed a priori that monotherapy with levodopa, dopamine agonists, catechol-O-methyl transferase (COMT) inhibitors, monoamine oxidase B (MAOB) inhibitors and anticholinergics all have evidence of efficacy with motor symptoms, at the expense of side effects. ${ }^{21-25}$ The evidence is mostly from short term studies so longer term efficacy and adverse effects are uncertain. There is no good evidence regarding the optimal time for treatment initiation or dosage increase. A recent very large randomised controlled trial did show very small but persistent benefits for patient-rated mobility scores when treatment is initiated with levodopa compared with levodopa-sparing therapy. ${ }^{26}$

For the treatment of motor complications with adjuvant therapies, the evidence supports that levodopa plus dopamine agonists, or COMT-inhibitors or MAOBinhibitors all reduce patients' off-time, reduce the required L-dopa dose, and improve motor and activities 
of daily living scores in PwP with motor complications on L-dopa. ${ }^{27}$ Again, the evidence is mostly from short term studies so longer term efficacy and adverse effects are uncertain. Which adjuvant drug is best is mostly uncertain, although the MAOB-inhibitor tolcapone overall has greater efficacy than entacapone but is associated with a worse adverse event profile. For the small subset of $\mathrm{PwP}$ able to tolerate deep brain stimulation (DBS) (estimated at $1-10 \%),{ }^{28}$ DBS to the subthalmic nucleus improves self-reported quality of life and reduces motor complications of PD up to 2 years when compared to best treatment with medications but at a higher risk of severe adverse events. ${ }^{29}$ Physiotherapy has short term benefits in PD (up to 3 months), but there is no evidence regarding the best sort of physiotherapy. ${ }^{30}$ Unfortunately there is insufficient research evidence for any 'certainties' in speech therapy ${ }^{31} 32$ and occupational therapy $^{33}$ for PD.

As a result of checking the uncertainties against current evidence we identified evidence to refute a number of submissions. One respondent suggested that ethnicity may affect the response to medications; ${ }^{22}{ }^{27}$ however anti-Parkinson's medications have been tested worldwide and no differences in efficacy or safety have been noted in relation to ethnicity. Another respondent suggested that immunosuppression was a side-effect of rasagaline; this is not a side effect that has ever been reported for rasagaline. ${ }^{34}$

As can be seen there are relatively few evidential certainties to inform the day-to-day management of PD.

\section{Prioritisation}

Eighteen uncertainties were excluded from the prioritisation by the steering group (although they were still entered onto the UK DUETS database) as they had less than three duplicate submissions and were deemed to be unlikely to be important enough to reach the top 10 priorities. Those statements that did reach the final top 10 had a range of 20-83 duplicate submissions.

The 94 uncertainties were then sent to the 409 participants that had provided their contact details, 302 members of the Research Support Network, and respondents to the magazine article and social media. The 94 uncertainties were prioritised by 475 participants consisting of $342 \mathrm{PwP} ; 57$ carers; 34 friends and family; and 42 health and social care professionals. The top 26 priorities of the four groups were summated, ranked and labelled $\mathrm{A}-\mathrm{Z}$ and presented to the final prioritisation workshop (table 2).

The final prioritisation workshop to agree the top 10 research priorities (table 3) had 27 participants including 10 PwP, 5 carers and family, 5 consultants, 4 PD nurse specialists and 3 allied health professionals. One word change was allowed on the fourth uncertainty; where it was changed from treatments being 'tailored' to 'developed' to suit phenotypes of PD. This was agreed with KHOD who had read all of the original responses that had generated this uncertainty and felt that this change of wording was still representative of the original respondents' intent. Although proposals were made to combine uncertainties, these were resisted as it was felt that this would have made the scope of the research questions too broad.

\section{DISCUSSION}

This study has identified the paucity of evidence currently available to address the everyday practicalities of managing a complex disease such as PD. The top 10 research priorities (table 3) included the need to address motor symptoms (balance and falls, and fine motor control), non-motor symptoms (sleep and urinary dysfunction), mental health issues (stress and anxiety, dementia, and mild cognitive impairments), side effects of medications (dyskinesia) and the need to develop interventions specific to the phenotypes of PD and better monitoring methods. These results will help funders identify future priorities for research that have greatest relevance to patients and the clinicians that treat them.

The fact that research around balance and falls was the top priority underscores the frequency of falls in PwP and the impact falls and fear of falling can have on more global issues such as function, quality of life and care home admission. ${ }^{35}$ Exercise can improve balance, but reducing falls is more challenging. ${ }^{36}{ }^{37}$ Although medications can improve overall motor performance which may reduce risk factors for falls, balance and falls are rarely measured or reported specifically in medication trials. ${ }^{22} 27$ Additionally there may be problems accessing appropriately trained physiotherapists ${ }^{38} 39$ and poor medication adherence ${ }^{40} 41$ may impact on effectiveness. Therefore there is a great need for research for effective interventions to improve balance and reduce falls in PwP.

\section{Specificity of research questions}

One criticism of Priority Setting Partnerships is that they generate research questions that are too broad and vague to inform researchable questions and funder priorities. ${ }^{13}$ We ensured the uncertainties in this study were as specific as possible, and did not allow similar uncertainties to be merged. Each uncertainty was informed by a number of initial statements, and each one was phrased so as to represent their overall intent. Therefore the use of the term 'treatments' was intended to cover a wide range of specific interventions such as pharmacological, behavioural and rehabilitation interventions.

Sometimes the lack of specificity of the question highlights the general lack of evidence around the issue. For example, the 9th uncertainty, 'improving dexterity', might be addressed by current medications but this is rarely recorded as an outcome in clinical trials. ${ }^{22} 27$ Occupational therapy interventions might be helpful for specific issues, for example, adapted computer mice, but there is very limited research in this area. ${ }^{33} 42$ We are 
What treatments are helpful in reducing tremor in people with Parkinson's?

What treatments are helpful for reducing balance problems and falls in people with Parkinson's?

Is it possible to identify different types of Parkinson's, eg, tremor dominant? And can we tailor

treatments best according to these different types?

What treatments would ensure the medications were equally effective each day (prevented/managed

wearing off, variability, on/off states) in people with Parkinson's?

Would the monitoring of dopamine levels in the body (eg, with blood tests) be helpful in determining

medication timing and amount (dose)?

What is helpful for improving the quality of sleep in people with Parkinson's?

What best treats mild cognitive problems such as memory loss, lack of concentration, indecision and

slowed thinking in people with Parkinson's?

What treatments are helpful in reducing urinary problems (urgency, irritable bladder, incontinence) in

people with Parkinson's?

What drug treatments are best for the different stages of Parkinson's?

What approaches are helpful for reducing stress and anxiety in people with Parkinson's?

What treatments are helpful for reducing dyskinesias (involuntary movements, which are a side effect

of some medications) in people with Parkinson's?

What best treats dementia in people with Parkinson's?

What interventions are effective for reducing or managing unexplained fatigue in people with

Parkinson's?

What best helps prevent or reduce freezing (of gait and in general) in people with Parkinson's?

What treatments are helpful for swallowing problems (dysphagia) in people with Parkinson's?

What is the best method of monitoring a person with Parkinson's response to treatments?

What training, techniques or aids are needed for hospital staff, to make sure patients with Parkinson's

get their medications correctly and on time?

What treatments are helpful in reducing bowel problems (constipation, incontinence) in people with

Parkinson's?

What is the best type and dose of exercise (physiotherapy) for improving muscle strength, flexibility,

fitness, balance and function in people with Parkinson's?

Can medications be developed to allow fewer doses per day for people with Parkinson's? (For

example combinations of medications in one pill, slow release pills)

What helps improve the dexterity (fine motor skills or coordination of small muscle movements) of

people with Parkinson's so they can do up buttons, use computers, phones, remote controls etc?

What treatments are effective in reducing hallucinations (including vivid dreams) in people with

Parkinson's?

What is the best treatment for stiffness (rigidity) in people with Parkinson's?

At which stage of Parkinson's is deep brain stimulation (a surgical treatment that involves implanting a

'brain pacemaker' that sends signals to specific parts of the brain) most helpful?

What training to improve knowledge and skills do informal carers (family and friends) need in order to

best care for people with Parkinson's?

What is the best treatment for pain in people with Parkinson's?

F\&F, family and friends; HSCP, health and social care professionals; PwP, people with Parkinson's.

\begin{tabular}{|c|c|c|c|c|c|c|}
\hline $\begin{array}{l}\text { PwP } \\
\text { Score }\end{array}$ & $\begin{array}{l}\text { Carer } \\
\text { Score }\end{array}$ & $\begin{array}{l}\text { F\&F } \\
\text { Score }\end{array}$ & $\begin{array}{l}\text { HSCP } \\
\text { Score }\end{array}$ & Total & $\begin{array}{l}\text { Interim } \\
\text { rank }\end{array}$ & $A-Z$ ID \\
\hline 93 & 83 & 92 & 91 & 359 & 1 & $\mathrm{~T}$ \\
\hline 92 & 93 & 80 & 94 & 359 & 1 & $E$ \\
\hline 88 & 88 & 89 & 88 & 353 & 3 & $U$ \\
\hline 89 & 94 & 88 & 81 & 352 & 4 & $\mathrm{H}$ \\
\hline 91 & 89 & 86 & 86 & 352 & 4 & L \\
\hline 94 & 79.5 & 93 & 84 & 350.5 & 6 & $\mathrm{G}$ \\
\hline 87 & 91 & 77 & 89.5 & 344.5 & 7 & D \\
\hline 90 & 77 & 94 & 79 & 340 & 8 & 1 \\
\hline 83 & 87 & 87 & 77.5 & 334.5 & 9 & $x$ \\
\hline 75 & 77 & 82 & 92 & 326 & 10 & $M$ \\
\hline 80 & 90 & 73.5 & 77.5 & 321 & 11 & $\mathrm{~J}$ \\
\hline 56 & 92 & 75 & 93 & 316 & 12 & Z \\
\hline 78 & 65 & 85 & 85 & 313 & 13 & $Y$ \\
\hline 79 & 71.5 & 76 & 82 & 308.5 & 14 & 0 \\
\hline 66 & 74.5 & 81 & 80 & 301.5 & 15 & C \\
\hline 81 & 52.5 & 83.5 & 83 & 300 & 16 & $\mathrm{~F}$ \\
\hline 53 & 86 & 64.5 & 89.5 & 293 & 17 & W \\
\hline 77 & 85 & 90 & 40 & 292 & 18 & $\mathrm{~K}$ \\
\hline 84 & 68 & 64.5 & 67.5 & 284 & 19 & $Q$ \\
\hline 73 & 84 & 56 & 69 & 282 & 20 & $S$ \\
\hline 85 & 59.5 & 73.5 & 54.5 & 272.5 & 21 & A \\
\hline 52 & 79.5 & 71.5 & 61 & 264 & 22 & $P$ \\
\hline 86 & 67 & 63 & 46 & 262 & 23 & $B$ \\
\hline 69 & 59.5 & 91 & 42 & 261.5 & 24 & $N$ \\
\hline 42 & 82 & 70 & 63.5 & 257.5 & 25 & V \\
\hline 82 & 54 & 60.5 & 57.5 & 254 & 26 & $\mathrm{R}$ \\
\hline
\end{tabular}


Table 3 Final prioritised and ranked uncertainties for the management of Parkinson's disease

Overarching research aspiration: an effective cure for Parkinson's disease

$1 \quad$ What treatments are helpful for reducing balance problems and falls in people with Parkinson's?

2 What approaches are helpful for reducing stress and anxiety in people with Parkinson's?

3 What treatments are helpful for reducing dyskinesias (involuntary movements, which are a side effect of some medications) in people with Parkinson's?

4 Is it possible to identify different types of Parkinson's, eg, tremor dominant? And can we develop treatments to address these different types?

5 What best treats dementia in people with Parkinson's?

6 What best treats mild cognitive problems such as memory loss, lack of concentration, indecision and slowed thinking in people with Parkinson's?

7 What is the best method of monitoring a person with Parkinson's response to treatments?

8 What is helpful for improving the quality of sleep in people with Parkinson's?

9 What helps improve the dexterity (fine motor skills or coordination of small muscle movements) of people with Parkinson's so they can do up buttons, use computers, phones, remote controls etc?

10 What treatments are helpful in reducing urinary problems (urgency, irritable bladder, incontinence) in people with Parkinson's?

even unsure of the impact of poor fine motor control and what assessments would best measure not only the amelioration of the impairment but improvements in activities and participation.

\section{Links with other research priority projects}

A Dutch study ${ }^{43}$ recently tried to identify patient-relevant research topics by interviews and focus groups with 57PwP, carers and researchers. These were then prioritised by $1360 \mathrm{PwP}$. The topics covered all areas of PD research including cure, diagnosis, psychological aspects, relationships and healthcare. The research topics identified were broad and their prioritisation unclear. Overall they reported that research into effective strategies for living and coping with the disease were the priority of PwP. Many of the top 10 priorities identified in this project could be said to address the need for effective strategies for living and coping with $\mathrm{PD}{ }^{43}$ Priority Setting Partnerships have been previously conducted for dementia and urinary incontinence. ${ }^{44}{ }^{45}$ Although some of the priorities from these projects might have relevance to $\mathrm{PwP}$ they did not prioritise Parkinson's-specific aspects of these conditions. This is almost certainly because the population of $\mathrm{PwP}$ with these problems is relatively small compared to the overall populations with these issues.

\section{Risk management}

This project raised a number of risk management challenges which should be considered in the design of future Priority Setting Partnerships.

We were only able to follow-up safeguarding issues where participants had provided their contact details. One response raised concerns about suicidality, and as we did not have the contact details for this participant, we had no way to contact the participant to ensure they had appropriate support. Future Priority Setting Partnerships should consider making the provision of contact details mandatory for participants so that issues like this can be followed up and support offered. However we recognise that this may inhibit participation, so it may be considered sufficient to provide participants with links to appropriate sources of support.

A number of other responses raised concerns about potential abuse (of both PwP and their carers), lack of appropriate service provision, and families failing to cope. We consulted with safeguarding experts who advised that safeguarding referrals were not appropriate or necessary but we responded to these participants and ensured they were aware of the support provided by Parkinson's UK. It would be best practice in future to let participants know in advance that if their responses cause concern for the research team there is likely to be some form of follow-up.

In response to the first survey, a couple of patients admitted to taking 'medication holidays' or adhering to their medication regime erratically (eg, every other day) and it was clear that these patients were unaware of the risks associated with this. ${ }^{46}$ As a result of this finding, Parkinson's UK are improving their information leaflets on this issue.

Finally, potential mental health needs of the research team transforming the responses into PICO questions and possible needs for support should be considered. Some of the responses described distressing situations and team members need to feel that they can discuss issues that concern them, and take breaks when needed in order to manage this stress and to obtain appropriate levels of support.

\section{Study limitations}

Although great efforts were made to include participants from black and minority ethnic and care home populations we were not very successful at recruiting these populations. It is also unlikely that those with literacy issues would participate in a project like this. Most 
respondents with PD were likely to be members of charities whose membership tends to be white, middle class and to have higher levels of education. Therefore the study results are more likely to be relevant to white PwP who live in their own homes either independently or with some assistance. This means that priorities of relevance to people with more severe disease (either palliative stage PD or with significant comorbidities) or to ethnic minorities may not have been identified, or if identified not given the priority that these populations would have given if fully represented in this exercise. Consideration should be given to identify the research priorities of these groups separately.

Another limitation was the relatively small proportion of social care professionals $(1 \%)$ who participated in this study. It is possible that as this professional group works mainly outside of healthcare settings they could have raised different unique research uncertainties.

Theoretically, the exclusion of statements with less than three duplicate submissions could have introduced bias. However by keeping the survey as short as possible we enhanced its accessibility. Also those statements that did reach the final top 10 had a range 20-83 duplicate submissions, so it is unlikely that a statement with less than three duplicate submissions would have reached the final top 10 .

\section{CONCLUSIONS}

This top 10 list of research priorities for the management of PD was generated using a systematic, transparent and inclusive method. The research priorities covered a wide range of topics of importance to those affected by the impact of PD; motor symptoms (balance and falls, and fine motor control), non-motor symptoms (sleep and urinary dysfunction), mental health issues (stress and anxiety, dementia, and mild cognitive impairments), side effects of medications (dyskinesia) and the need to develop interventions specific to the phenotypes of PD and better monitoring methods. It is hoped that the findings will lead to future research that will address issues of importance for the clinical management of PD.

Acknowledgements The authors would like to thank everyone who submitted uncertainties and prioritised them, the members of the steering group, Katherine Cowan of JLA, Mark Fenton of UK DUETs and all the staff and volunteers at Parkinson's UK who facilitated access to the project and inputted the hard copy versions of the surveys. The Norwich Clinical Trials Unit provided expert database design and support.

Contributors KHOD and HF prepared the first version of the manuscript. DJD, $\mathrm{RP}, \mathrm{KHOD}$ and HF transformed responses into PICO questions. CEC and CS provided expert clinical research advice. BP provided expert safeguarding advice. SS oversaw the data collection and input. All authors reviewed the manuscript and edited it for content and interpretation.

Funding This work was supported by Parkinson's UK.

Competing interests CEC received honoraria for lectures, travel expenses for conferences, and unrestricted educational grants from AbbVie, Britannia, Teva-Lundbeck, and UCB.

Ethics approval University of East Anglia, Research Ethics Committee.
Provenance and peer review Not commissioned; externally peer reviewed.

Data sharing statement All surveys are available in online supplementary materials. The anonymised dataset of the 4100 responses will be available from Parkinson's UK. The 108 uncertainties will be available from UK DUETS.

Open Access This is an Open Access article distributed in accordance with the Creative Commons Attribution Non Commercial (CC BY-NC 4.0) license, which permits others to distribute, remix, adapt, build upon this work noncommercially, and license their derivative works on different terms, provided the original work is properly cited and the use is non-commercial. See: http:// creativecommons.org/licenses/by-nc/4.0/

\section{REFERENCES}

1. Delaney $B$. Is society losing control of the medical research agenda? BMJ 2006;332:1063-4.

2. Tallon D, Chard J, Dieppe P. Relation between agendas of the research community and the research consumer. Lancet 2000;355:2037-40.

3. Patsopoulos NA, lonnidis JPA, Analatos AA. Origin and funding of the most frequently cited papers in medicine: database analysis. BMJ 2006;332:1061-4.

4. Chalmers I. Well informed uncertainties about the effects of treatments: how should clinicians and patients respond? BMJ 2004;328:475-6.

5. Partridge N, Scadding J. The James Lind Alliance: patients and clinicians should jointly identify their priorities for clinical trials. Lancet 2004;364:1923-4.

6. Thornton $\mathrm{H}$. Patient and public involvement in clinical trials. BMJ 2008;336:903-4.

7. Chalmers I. Confronting therapeutic ignorance. BMJ 2008;337:a84.

8. Core Outcome Measures in Effectiveness Trials Initiative (COMET). http://www.comet-initiative.org/ (accessed 4 Aug 2014).

9. Consensus-based Standards for the selection of health Measurement Instruments (COSMIN). http://www.cosmin.nl/ (accessed 4 Aug 2014).

10. DUETS. Identifying and prioritising unanswered questions about the effects of treatment: the role of the Database of Uncertainties about the Effects of Treatments (DUETs) and the James Lind Alliance. The Database of Uncertainties about the Effects of Treatments (DUETs). 2006 http://www.library.nhs.uk/duets/ (accessed 28 Jul 2014).

11. The James Lind Alliance: 2004. http://www.lindalliance.org/ (accessed 28 Jul 2014).

12. Cowan K, Oliver S. The James Lind Alliance guidebook. Version 5 Oxford: James Lind Alliance, 2013. http://www.JLAguidebook.org (accessed 6 Aug 2014).

13. NICE/AMRC/JLI. Research prioritisation. Report of a meeting held in London on 28 January. 2013. http://www.lindalliance.org/pdfs/ Publications/2013_JLI-NICE-AMRC_RtableReport_Research_ Prioritisation.pdf (accessed 4 Aug 2014).

14. Parkinson's UK research strategy 2010-2014. 2010. London: Parkinson's UK. http://www.parkinsons.org.uk/sites/default/files/ publications/download/english/researchstrategy2010_2014_0.pdf (accessed 4 Aug 2014).

15. Parkinson's UK Research Support Network. http://www.parkinsons. org.uk/content/research-support-network (accessed 20 Aug 2014).

16. National Research Ethics Service (NRES). http://www.nres.nhs.uk/ (accessed 4 Aug 2014).

17. The NHS constitution. 2013. London: The Department of Health. http://www.nhs.uk/choiceintheNHS/Rightsandpledges/ NHSConstitution/Pages/Overview.aspx (accessed 6 Aug 2014)

18. Tracking Parkinson's Project. http://www.parkinsons.org.uk/content/ tracking-parkinsons (accessed 13 Aug 2014).

19. Dementias and Neurodegeneration Specialty Clinical Research Network (DeNDRoN). http://www.crn.nihr.ac.uk/dementia/ (accessed 13 Aug 2014).

20. Enabling Research in Care Homes (ENRICH). http://www.enrich. dendron.nihr.ac.uk/ (accessed 4 Aug 2014).

21. van Hilten JJ Ramaker CC, Stowe R, et al. Bromocriptine/levodopa combined versus levodopa alone for early Parkinson's disease. Cochrane Database Syst Rev 2007;(4):CD003634.

22. Stowe R, Ives N, Clarke CE, et al. Dopamine agonist therapy in early Parkinson's disease. Cochrane Database Syst Rev 2008;(2): CD006564.

23. Deane K, Spieker S, Clarke CE. Catechol-O-methyltransferase inhibitors versus active comparators for levodopa-induced complications in Parkinson's disease. Cochrane Database Syst Rev 2004;(4):CD004553. 
24. Caslake R, Macleod A, Ives N, et al. Monoamine oxidase B inhibitors versus other dopaminergic agents in early Parkinson's disease. Cochrane Database Syst Rev 2009;(4):CD006661.

25. Katzenschlager R, Sampaio C, Costa J, et al. Anticholinergics for symptomatic management of Parkinsońs disease. Cochrane Database Syst Rev 2002;(3):CD003735.

26. Gray R, Ives N, Rick C; PD MED Collaborative Group. Long-term effectiveness of dopamine agonists and monoamine oxidase $B$ inhibitors compared with levodopa as initial treatment for Parkinson's disease (PD MED): a large, open-label, pragmatic randomised trial. Lancet 2014;384:1196-205. http://dx.doi.org/10.1016/S0140-6736 (14)60683-8

27. Stowe RL, Ives NJ, Clarke CE, et al. Meta-analysis of the comparative efficacy and safety of adjuvant treatment to levodopa in later Parkinson's disease. Mov Disord 2011:26:587-98.

28. Nicholson T, Milne R. Pallidotomy, thalamotomy and deep brain stimulation for severe Parkinson's disease. Southampton: Wessex Institute for Health Research and Development, 1999 December. Development and Evaluation Committee Report No. 105.

29. NHS Commissioning Board Clinical Reference Group for Adult Neurosurgery. Clinical Commissioning Policy: Deep Brain Stimulation (DBS) in Movement Disorders (Parkinson's Disease, Tremor and Dystonia). 2013. http://www.england.nhs.uk/wp-content/ uploads/2013/04/d03-p-b.pdf (accessed 4 Aug 2014).

30. Tomlinson CL, Patel S, Meek C, et al. Physiotherapy intervention in Parkinson's disease: a systematic review and meta-analysis of the randomised controlled trials. BMJ 2012;345:e5004

31. Herd C, Tomlinson CL, Deane KHO, et al. Speech and language therapy versus placebo or no intervention for speech problems in Parkinson's disease. Cochrane Database Syst Rev 2012;(8): CD002812.

32. Herd CP, Tomlinson CL, Deane KHO, et al. Comparison of speech and language therapy techniques for speech problems in Parkinson's disease. Cochrane Database Syst Rev 2012;(8):CD002814.

33. Dixon L, Duncan D, Johnson P, et al. Occupational therapy for patients with Parkinson's disease. Cochrane Database Syst Rev 2007;(3):CD002813.

34. Chen JJ, Swope DM, Dashtipour K. Comprehensive review of rasagiline, a second-generation monoamine oxidase inhibitor, for the treatment of Parkinson's disease. Clin Ther 2007;29:1825-49.
35. Cumming RG, Salkeld G, Thomas M, et al. Prospective study of the impact of fear of falling on activities of daily living, SF-36 scores, and nursing home admission. J Gerontol A Biol Sci Med Sci 2000;55A: M299-305.

36. Pickering RM, Grimbergen YAM, Rigney $\mathrm{U}$, et al. A meta-analysis of six prospective studies of falling in Parkinson's disease. Mov Disord 2007;22:1892-900

37. Allen NE, Sherrington C, Paul SS, et al. Balance and falls in Parkinson's disease: a meta-analysis of the effect of exercise and motor training. Mov Disord 2011;26:1605-15.

38. Parkinson's UK. National Parkinson's Audit Report 2012. London: Parkinson's UK, 2013 https://www.parkinsons.org.uk/sites/default/ files/nationalparkinsonsaudit2012.pdf (accessed 7 Aug 2014).

39. Parkinson's Disease Society. Life with Parkinson's today-room for improvement. London: Parkinson's Disease Society. 2008. http:// www.parkinsons.org.uk/content/ life-parkinsons-today-room-improvement (accessed 7 Aug 2014).

40. Daley DJ, Myint PK, Gray RJ, et al. Systematic review on factors associated with medication non-adherence in Parkinson's disease. Parkinsonism Relat Disord 2012;18:1053-61.

41. Daley DJ, Deane KHO, Gray RJ, et al. Adherence therapy improves medication adherence and quality of life in people with Parkinson's disease: a randomised controlled trial. Int J Clin Pract 2014;68:963-71.

42. Sturkenboom IH, Graff MJ, Borm GF, et al. The impact of occupational therapy in Parkinson's disease: a randomized controlled feasibility study. Clin Rehabil 2013;27:99-112.

43. Schipper K, Dauwerse L, Hendrikx A, et al. Living with Parkinson's disease: priorities for research suggested by patients. Parkinsonism Relat Disord 2014;20:862-6.

44. Outcomes of the James Lind Alliance Dementia priority setting partnership. Alzheimer's Society. 2013. http://alzheimers.org.uk/site/ scripts/download_info.php?fileID=2226 (accessed 4 Aug 2014).

45. Buckley BS, Grant AM, Tincello DG, et al. Prioritizing research: patients, carers, and clinicians working together to identify and prioritize important clinical uncertainties in urinary incontinence. Neurourol Urodyn 2010;29:708-14.

46. NICE. Parkinson's disease: Diagnosis and management in primary and secondary care. 2006:CG35. http://www.nice.org.uk/guidance/ cg35 (accessed 4 Aug 2014). 